\title{
Antibody secreting cells are critically dependent on integrin a4 $37 /$ MAdCAM-1 for intestinal recruitment and control of the microbiota during chronic colitis
}

\author{
Christopher J. Tyler ${ }^{1,2}$, Mauricio Guzman ${ }^{1,2}$, Luke R. Lundborg ${ }^{1,2}$, Shaila Yeasmin ${ }^{1,2}$, Nadia Zgajnar ${ }^{1,2}$, Paul Jedlicka ${ }^{3}$, \\ Giorgos Bamias ${ }^{4}$ and Jesús Rivera-Nieves ${ }^{1,2}{ }^{凶}$
}

This is a U.S. government work and not under copyright protection in the U.S.; foreign copyright protection may apply 2021

$T$ and B cells employ integrin a $4 \beta 7$ to migrate to intestine under homeostatic conditions. Whether those cells differentially rely on a4 $\beta 7$ for homing during inflammatory conditions has not been fully examined. This may have implications for our understanding of the mode of action of anti-integrin therapies in inflammatory bowel disease (IBD). Here, we examined the role of a4 $\beta 7$ integrin

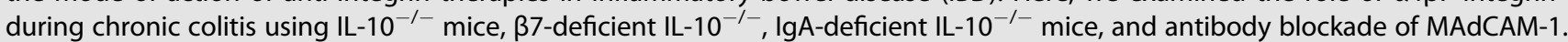
We found that $\alpha 4 \beta 7$ was predominantly expressed by B cells. $\beta 7$ deficiency and MAdCAM- 1 blockade specifically depleted antibody secreting cells (ASC) (not T cells) from the colonic LP, leading to a fecal pan-immunoglobulin deficit, severe colitis, and alterations of microbiota composition. Colitis was not due to defective regulation, as dendritic cells (DC), regulatory $T$ cells, retinaldehyde dehydrogenase (RALDH) expression, activity, and regulatory T/B-cell cytokines were all comparable between the strains/treatment. Finally, an IgA deficit closely recapitulated the clinical phenotype and altered microbiota composition of $\beta 7-$ deficient IL-10 ${ }^{-/-}$mice. Thus, a luminal IgA deficit contributes to accelerated colitis in the $\beta 7$-deficient state. Given the critical/nonredundant dependence of IgA ASC on a4ß7:MAdCAM-1 for intestinal homing, B cells may represent unappreciated targets of anti-integrin therapies.

Mucosal Immunology (2022) 15:109-119; https://doi.org/10.1038/s41385-021-00445-z

\section{INTRODUCTION}

Maintenance of a homeostatic microbiota is critically dependent on antibody secreting cells (ASC) which release IgA to maintain a balance between the microbiota and its host ${ }^{1}$. Loss of tolerance to antigens derived from the intestinal microbiota has been implicated in the pathogenesis of inflammatory bowel disease (IBD). Yet little effort has been placed on understanding the role of $\mathrm{B}$ cells/lgA during the pathogenesis of IBD, particularly considering that about $80 \%$ of the body's ASC are found in the intestine. Lymphocytes in general and B cells in particular are imprinted with a gut-homing program within inductive sites (e.g., Peyer patches (PP), mesenteric lymph nodes (MLN)) by upregulating a4 $\beta 7$ integrin expression mediated by retinoic acid (RA)-producing dendritic cells $(D C)^{2}$. This enables homing to the intestine and gastrointestinal-associated lymphoid tissues (GALT) through specialized venules that express the a4 $\beta 7$ ligand: mucosal addressin cell adhesion molecule-1 (MAdCAM-1). MAdCAM-1 is an endothelial adhesion molecule of the immunoglobulin superfamily expressed by postcapillary venules of the small and large intestinal lamina propria (LP), MLN, and mammary gland ${ }^{3}$.

A critical dependence of $B$ cells/ASC on a4ß7/MAdCAM-1 interactions for intestinal homing is supported by the fact that $\operatorname{lgA}^{+}$ASC are decreased in both 37 - and MAdCAM-1-deficient mice ${ }^{4,5}$. However, whether there may be differential dependence by $\mathrm{T}$ or B cells for intestinal recruitment has not been addressed. This is particularly relevant to understand the mode of action of drugs that targets this family of integrins in IBD, which is hallmarked by chronic dysregulated leukocyte recruitment. The integrin-antagonist vedolizumab ${ }^{6,7}$ (VDZ, anti-a4 $\beta 7$ ) is widely used for the treatment of IBD. Two additional drugs, etrolizumab (anti$\beta 7)$ and ontamalimab (anti-MAdCAM-1), are undergoing clinical trials $^{8}$. B cells and ASC express $\alpha 4 \beta 7^{9}$ and bind to the anti- $\alpha 4 \beta 7$ antibody VDZ at equal or higher levels than T cells ${ }^{10}$. A commonly held premise is that VDZ is intestinal specific, and acts mainly by blocking effector T-cell entry to the intestinal LP. However, there is insufficient evidence for this from human data ${ }^{11}$, where minimal effect on LP T-cell populations has been observed, implicating innate immune cells, namely macrophages and $\mathrm{DC}^{12}$ and monocytes ${ }^{13}$. Clinically, less than $50 \%$ of IBD patients who are treated with VDZ achieve long-term clinical remission, and an even smaller fraction meets the more demanding treatment targets of mucosal, histological or deep remission ${ }^{6,7}$. These shortcomings emphasize the need to clearly define its mechanism of action to better identify patients with increased probability for response ${ }^{14}$. In fact, despite widespread use of VDZ for more than 6 years, the precise cell targets which define its anti-inflammatory effects remain uncertain ${ }^{11,14}$.

\footnotetext{
${ }^{1}$ San Diego VA Medical Center, San Diego, CA, USA. ${ }^{2}$ Division of Gastroenterology, University of California San Diego, La Jolla, CA, USA. ${ }^{3}$ Department of Pathology, University of Colorado Denver, Denver, CO, USA. ${ }^{4}$ GI Unit, 3rd Academic Department of Internal Medicine, Sotiria Hospital, National and Kapodistrian University of Athens, Athens, Greece.

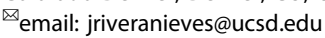


a

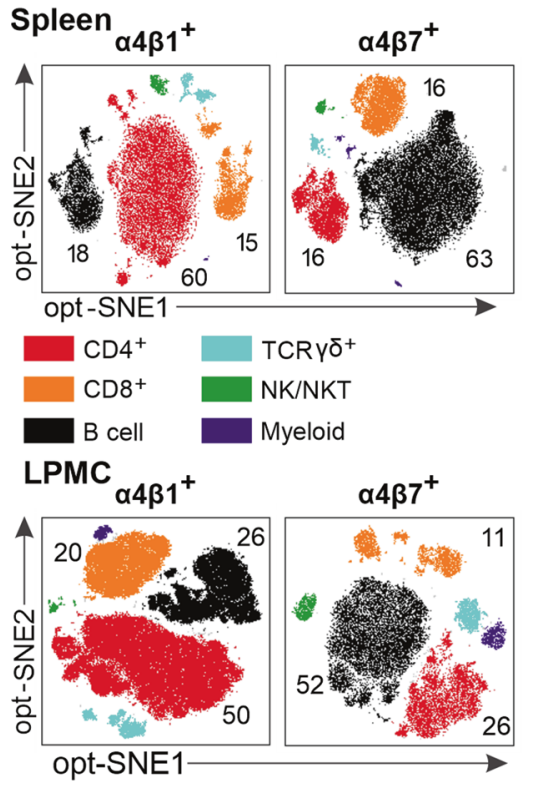

b

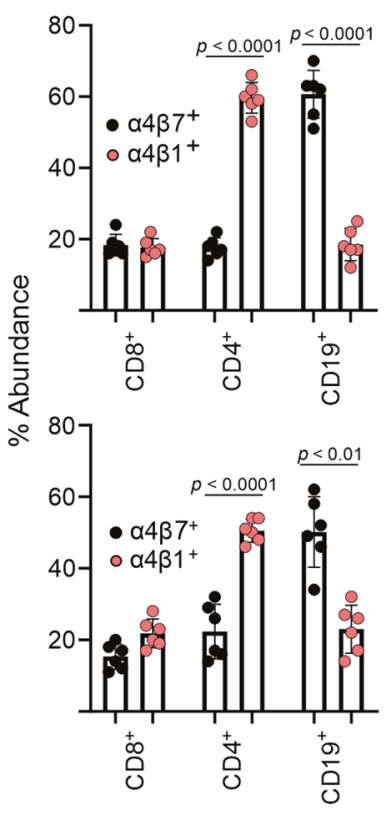

C

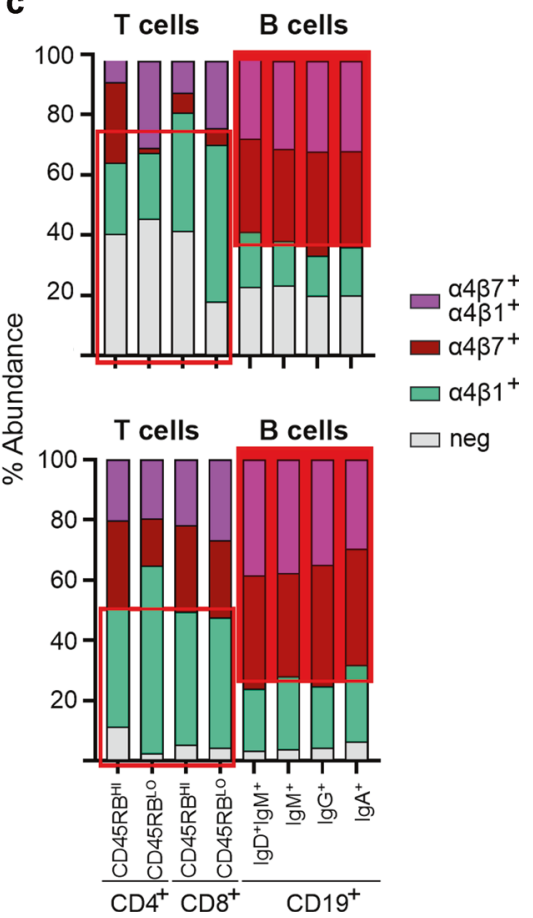

Fig. 1 B cells predominantly express a $4 \beta 7$ integrin. a Cellular distribution of integrins $\alpha 4 \beta 1$ and $\alpha 4 \beta 7$ among the major leukocyte lineages within the peripheral (spleen) and mucosal (colonic LP) compartments. Cells were pregated on live, CD45 ${ }^{+}$cells, followed by opt-SNE analysis. Cell populations including $\mathrm{CD}^{+}\left(\mathrm{CD}^{+} \mathrm{T}\right.$ cells), $\mathrm{CD}^{+}\left(\mathrm{CD}^{+} \mathrm{T}\right.$ cells $), \mathrm{CD} 19^{+} / \mathrm{lgA}^{+} / \mathrm{lgG}^{+}$(B cells), TCR $\gamma \delta^{+}(\gamma \delta \mathrm{T}$ cells $), \mathrm{CD} 56^{+}(\mathrm{NK} / \mathrm{NKT})$, or $\mathrm{MHCII}^{+} / \mathrm{CD} 11 \mathrm{c}^{+} / \mathrm{CD} 11 \mathrm{~b}^{+}$(myeloid) cells are highlighted. b Percentages of $\alpha 4 \beta 1$ - and $\alpha 4 \beta 7$-expressing cells within the indicated cell lineages from colonic LPMC and splenocytes. Each data point represents a single mouse. All data are presented as mean \pm S.D, from $n>6$ mice in each dataset. Statistical significance determined using ANOVA, followed by Tukey's multiple comparison test. c Distribution of integrin expressing subpopulations within the $\mathrm{CD}^{+}{ }^{+} \mathrm{CD}^{+}$, and B-cell lineages. T- and B-cell populations were divided into the indicated memory subpopulations and the expression of $\alpha 4 \beta 7$ and $\alpha 4 \beta 1$ integrins determined.

We set out to evaluate the immune cell lineages most reliant on integrin $\alpha 4 \beta 7$ to enter the intestine during chronic inflammatory conditions, using the IL-10 $10^{-/-}$murine model of chronic colitis. IL10 deficient mice develop spontaneous colitis that shares features with ulcerative colitis (UC), marked by epithelial cell hyperplasia and transmural inflammation. The microbiota plays a major role in this model ${ }^{15}$, as IL-10 $0^{-1-}$ germ-free mice do not develop colitis, whereas colonization by Helicobacter hepaticus triggers colitis ${ }^{16}$. We observed dichotomous a4 integrin expression by T and B cells, inasmuch the former express predominantly $\alpha 4 \beta 1$, and the latter a4 $\beta 7 . \mathrm{CD}^{+}$cells increased in colonic LP of $\beta 7$-sufficient and deficient colitic mice compared with noninflamed controls, whereas B cell/ASC populations did not change in $\beta 7$-deficient mice. The $B$ cell/ASC deficit in the LP resulted in a luminal panimmunoglobulin deficit and severe colitis, compared with $\beta 7$ sufficient mice. Colitis was not due to defective immune regulation, as DC, regulatory $\mathrm{T}$ cells (Tregs), RALDH expression, RALDH activity, and regulatory T/B-cell cytokines were all comparable between the strains. Furthermore, MAdCAM-1 blockade, which does not interfere with $a E \beta 7: E-c a d h e r i n$ interactions, impaired B cell/ASC recruitment specifically and recapitulated the colitic phenotype of $\beta 7$-deficient animals in real time. Finally, we identified $\lg A$ as the molecular effector for the accelerated colitis in the $\beta 7$-deficient state as IgA deficiency closely recapitulated the severe colitic phenotype, bacterial overgrowth, and alterations in fecal microbiota composition.

\section{RESULTS}

B cells preferentially express integrin $\alpha 4 \beta 7$

The $\alpha 4$ integrins ( $\alpha 4 \beta 1, a 4 \beta 7)$ are expressed by $T$ and $B$ cells ${ }^{3,9}$ and other leukocyte populations but whether certain cells may preferentially express either integrin heterodimer has not been demonstrated. Given potential redundancies of cell trafficking pathways during chronic inflammation ${ }^{17}$, we analyzed the surface expression of integrins $\alpha 4 \beta 1$ and $\alpha 4 \beta 7$ in the spleen, colonic LP, $\mathrm{MLN}$, and peripheral blood of IL-10 $0^{-1-}$ mice via mass cytometry. Our analysis demonstrated that T cells in spleen, LP, and blood predominantly expressed $\alpha 4 \beta 1$, while the B-cell lineage predominantly expressed $a 4 \beta 7^{+}$(Fig. 1a, b, Supplementary Fig. 1a, b). The MLN was the exception, where $C D 8^{+}$T cells were mostly $a 4 \beta 7^{+}$ and $B$ cells expressing $a 4 \beta 1^{+}$were most abundant (Supplementary Fig. 1a, b). We additionally examined the expression of a4 integrins on T-cell subsets $\left(\mathrm{CD} 4^{+} / \mathrm{CD} 25^{+}\right.$which include most Tregs, $\mathrm{CD} 4{ }^{+} \mathrm{CD} 44^{+}$effectors, $\mathrm{CD} 4^{+} / \mathrm{CD} 6 \mathrm{~L}^{+}$naive) as well as monocyte lineage cells $\left(\mathrm{CD} 11 \mathrm{c}^{+} / \mathrm{MHCl}^{+}\right.$and $\left.\mathrm{CD} 14^{+} / \mathrm{CD} 11 \mathrm{~b}^{+}\right)$. The three CD4 subsets and two myeloid lineage subsets examined predominantly express a4 $\beta 1$ (Supplementary Fig. 2). The derivation strategy for these analyses is shown in Supplementary Fig. 3a. To a lesser extent, other cell lineages also expressed $\alpha 4 \beta 7^{+}$, including $\mathrm{TCR} \gamma \delta^{+}$, natural killer (NK), and myeloid cells (Supplementary Fig. 3b). Surface integrin expression does not appear to change during inflammation, as it was not different in T or B cells isolated from inflamed and uninflamed IL-10 $10^{-1-}$ mouse colon (Supplementary Fig. 3c). In all, our findings support that most B cells in three of four immune sites examined predominantly express $\alpha 4 \beta 7$, whereas $\mathrm{CD}^{+}{ }^{+}$and some monocyte lineage cells express predominantly $a 4 \beta 1$, both in intestine and in the periphery.

\section{Integrin $\beta 7-$ deficient IL-10 ${ }^{-1-}$ mice develop severe lethal colitis, which is associated with deficits in colonic B cell/ASC numbers and fecal IgA/M content}

Since our initial findings pointed to a predominant expression of a $4 \beta 7$ by $B$ cells, we next examined its potential functional 
implications by assessing the effects of $\beta 7$ deficiency on the colitic phenotype of $\mathrm{IL}-10^{-/-}$mice. We compared the clinical phenotypes, immune cell distribution, and fecal Ig levels of $\beta 7$-sufficient (IL-10 $0^{-1-}$ ), with that of $\beta 7$-deficient mice

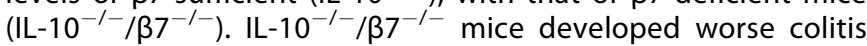
than their $\beta 7$-sufficient counterparts, as indicated clinically by lower body weights, hypothermia, and decreased survival (Fig. 2a, b), as well as the nearly uniform development of rectal prolapse (Supplementary Fig. 4a). In addition, $\mathrm{IL}-10^{-/-} / \beta 7^{-1-}$ mice had significantly higher histological indices of colitis (Fig. 2c, Supplementary Fig. 4b), while the ileum was mostly uninvolved (Supplementary Fig. 4c). In our colony, $\beta 7$-sufficient IL-10 $10^{-1-}$ mice do not exhibit spontaneous colitis until after 16 weeks of age, which is often manifested clinically by rectal prolapse. Tissues were harvested once $\beta 7$-deficient animals had lost $20 \%$ body weight (as per IRB regulations), which ranged from 7 to 12 weeks of age.

We next explored unexamined mechanisms that may underlie the increased severity of colitis in $\beta 7$-deficient mice, by comparing the cellular composition of the colonic LP between the two strains ( $\beta 7^{-1-}$ vs. $\beta 7^{+/+} / \mathrm{IL}-10^{-1-}$ mice). Given the variable penetrance of colitis in the strain, we separately analyzed noninflamed (N/I) and inflamed (I) mice, based on their histological colitis severity from 8 to 12 weeks of age, as determined by a pathologist in a blinded fashion, using a semiquantitative scoring system ${ }^{18}$. Our analysis showed that, upon development of intestinal inflammation, absolute numbers of both $\mathrm{CD}^{+}$and $\mathrm{CD}^{+} 9^{+}$cells increased in colitic (I) as compared with noncolitic (N/I) IL-10 ${ }^{-/-}$ mice (Fig. 2d, e). Further increase in $\mathrm{CD}^{+}$T-cell counts was seen in $\mathrm{IL}-10^{-/-} / \beta 7^{-/-}$mice corresponding to their more severe colitis (Fig. 2 d). However, $\mathrm{CD} 19^{+}$cell counts remained at baseline in IL-10 $0^{-/-} / \beta 7^{-1-}$ mice, despite their worse colitis (Fig. 2e). Further analysis revealed that the LP cell deficit was not limited to $\mathrm{CD}^{1} 9^{+}$B cells, but included $\operatorname{lgM}^{+}$and $\operatorname{lgA}^{+}$ASC (Fig. 2f-h). The LP ASC deficit was accompanied by lower fecal concentrations of immunoglobulins IgM, IgA (Fig. 2i), and lgG1-3 (Supplementary Fig. 4d), indicating a luminal pan-immunoglobulin deficit. T- and B-cell counts were mostly unchanged in spleen and MLN, in support of an intestinal-specific recruitment defect (Supplementary Fig. 4e). Taken together, these results suggest that influx of $B$-cell lineage cells into the inflamed intestinal mucosa is impaired in $\beta 7$-deficient IL- $10^{-1-}$ mice, whereas CD4 ${ }^{+}$T-cell entry remained unaffected. Therefore, $\beta 7$ integrin deficiency appeared to lead to selective inhibition of B-cell intestinal trafficking, which results in low numbers of ASC at the colonic LP and a corresponding deficit of intraluminal immunoglobulins. These immunological effects are associated with significant worsening of colitis in $\beta 7$-deficient mice, raising the possibility for a proinflammatory effect of the integrin deficit during the early stages of colitis induction, mediated by a fecal Ig deficit.

\section{Accelerated colitis in $\beta 7$-deficient mice is not due to failure of mucosal regulatory mechanisms}

Next, in order to understand the mechanisms behind the acceleration of colitis in IL-10 $10^{-1-} / \beta 7^{-1-}$ mice, we first tested the hypothesis that it was due to inadequate regulatory control at the intestinal mucosa. In fact, this was a plausible explanation, as $\beta 7^{-1-}$ mice, in addition to $\alpha 4 \beta 7$, lack $\alpha E(C D 103) / \beta 7$ integrin also, which is involved in regulatory mechanisms. In particular, CD103 is expressed by a subset of intestinal $\mathrm{DC}^{19}$ that produce $\mathrm{RA}$, and which are involved in the imprinting of a gut-homing phenotype on lymphocytes, induction of peripheral Tregs, and IgA class switching in $\mathrm{ASC}^{2}$. Accordingly, to test whether such regulatory mechanisms were defective in $\mathrm{IL}-10^{-1-} / \beta 7^{-1-}$ mice and responsible for the severe colitis phenotype, we measured the number of intestinal DC and Tregs, as well as the local production of Treg- or regulatory B-cell (Breg)-derived cytokines ${ }^{20}$ and the integrity of RA metabolism². Nevertheless, we did not observe a shortfall of either colonic CD11c ${ }^{+}$DC or Tregs (CD4/CD25/FoxP3 ${ }^{+}$) (Fig. 3a).
Similarly, aldefluor conversion, a surrogate indicator of retinaldehyde dehydrogenase (RALDH) activity (Fig. 3b), and RALDH1,2,3 mRNA expression (Fig. $3 \mathrm{C}$ ) were normal in the LP of $\mathrm{IL}-10^{-1-} / \beta 7^{-1-}$ mice. Finally, the levels of T/B-cell regulatory cytokines (TGF- $\beta$, and IL-35 subunits $\mathrm{EBI} 3$ and IL-12A) (Fig. 3d) were equally expressed in $\beta 7$ deficient and sufficient IL-10 $10^{-1-}$ mice. Taken together, these findings indicate that impaired regulatory mechanisms mediated by $\mathrm{CD}_{103}{ }^{+}$ $\mathrm{DC}$, Treg, or Bregs are preserved in $\mathrm{IL}-10^{-1-} / \beta 7^{-1-}$ mice and do not appear to play a major role on the aggravation of colitis, under the specific conditions of our IL-10 $10^{-/-}$mouse colony.

\section{MAdCAM-1 blockade worsened colitis in IL-10 ${ }^{-1-}$ mice in association with depletion of colonic B cells and ASC, deficit in fecal IgA content, and generation of a dysbiotic microbiota}

Mice that are deficient for $\beta 7$ have a functional impairment that involves both aE $\beta 7$ and $\alpha 4 \beta 7$ integrins, either of which may be responsible for the worsening of colitis observed in $\mathrm{IL}-10^{-1-} / \beta 7^{-1-}$ mice. Thus, in order to dissect the roles of aE $\beta 7$ and $a 4 \beta 7$ during chronic colitis, we treated IL-10 $10^{-1-}$ mice with an antibody against the a4 37 -specific ligand, MAdCAM-1 (MECA-367). In this way we specifically blocked a4 37 -dependent traffic, leaving aEß7-mediated pathways unaffected. Serial clinical and laboratory parameters were assessed prospectively and tissues were harvested after 8 weeks, when weights reached $80 \%$ of original at day 0 . Mass cytometry analysis of the colonic LP showed that $\mathrm{CD}^{+}$and $\mathrm{CD}^{+}$T-cell populations increased, whereas $\mathrm{CD}_{19}{ }^{+} \operatorname{lgM}^{+}$and $\lg \mathrm{A}^{+}$ASC were lower, compared with isotype-treated $\mathrm{IL}-10^{-/-}$mice (Fig. 4a, b). Conversely, the percentage of $\mathrm{CD}_{19} \operatorname{lgM}^{+}, \lg \mathrm{A}^{+}$, and $\operatorname{lgG}^{+} \mathrm{ASC}$ increased in the spleen, whereas $\mathrm{CD}^{+}$and $\mathrm{CD}^{+}$did not change (Supplementary Fig. 5a, b, e). Absolute numbers calculated with counting beads on a separate cohort using spectral cytometry confirmed these findings (Supplementary Fig. 5b). Clinically, mice developed progressive hypothermia starting at week 4 (Fig. 4c), followed by weight loss by week 6 (Fig. 4d). Inflammatory indices were higher in the colon and cecum of MECA-367-treated mice (Fig. 4e). Conversely, fecal IgA, IgM, and IgG levels decreased significantly by week 5 (Fig. 4f, Supplementary Fig. $5 f$ ) with a reverse increase in plasma $\lg \mathrm{A}, \lg \mathrm{M}$, and $\lg \mathrm{G}$ beginning after 2 weeks, which preceded all other clinical and laboratory changes (Fig. 4g, Supplementary Fig. $5 \mathrm{~g}$ ). In agreement, $\operatorname{lgA} / \mathrm{M}^{+} \mathrm{ASCs}$ and $\mathrm{CD} 19^{+}$B cells increased in peripheral blood after 3 weeks (Fig. 4h, Supplementary Fig. $5 c, d$ ), whereas $\mathrm{CD}^{+}$T-cell counts were unaffected (Supplementary Fig. 5e). In our hands, MECA-367 neither affected the percentages of Tregs nor regulatory T/B-cell-derived cytokines (TGF- $\beta$, EBI3, IL12-A) (Supplementary Fig. 5h, i). In a separate experiment antibody blockade of the a4 integrin, which blocks both integrins a4 $\beta 7$ and a $4 \beta 1$ also induced weight loss, hypothermia and colitis in IL- $10^{-1}$ mice (Supplementary Fig. 6). Ongoing studies will attempt to define the relative functional dependence on these integrins for induction and maintenance of chronic colitis.

A relatively stable phenotype of unperturbed $\mathrm{IL}-10^{-1-}$ mice, rendered defective in a4ß7:MAdCAM- 1 interactions either via $\beta 7$ deletion and anti-MAdCAM-1 antibody blockade led to a deficit of intraluminal IgA. Given the fact that IgA plays a crucial role for the maintenance of a homeostatic microbiota ${ }^{21}$, we hypothesized that inadequate control of the commensal microbiota leading to bacterial overgrowth/dysbiosis may contribute to the worsening of colitis. We, first, tested our hypothesis by examining fecal microbial counts and composition in cohoused IL-10 $10^{-1-}$ mice treated with MECA-367 or isotype antibody via $16 \mathrm{~S}$ qPCR and rRNA sequencing. Fecal bacterial counts were higher in MECA-367treated mice, with lower a diversity (Fig. $4 \mathrm{i}$ ) and altered $\beta$ diversity by principal component analysis (Fig. 4j, k) compared with isotype-treated controls. Taken together, we show that MAdCAM-1 and a4 blockade recapitulate changes observed in $\beta 7$-deficient mice thus, supporting a critical dependence of B cell/ ASC on a4ß7:MAdCAM-1 interactions for traffic to intestine and maintenance of luminal $\lg A$. 
a $\quad \mathrm{IL}-10^{-/-}$

- IL-10-1- $\beta 7^{-1-}$
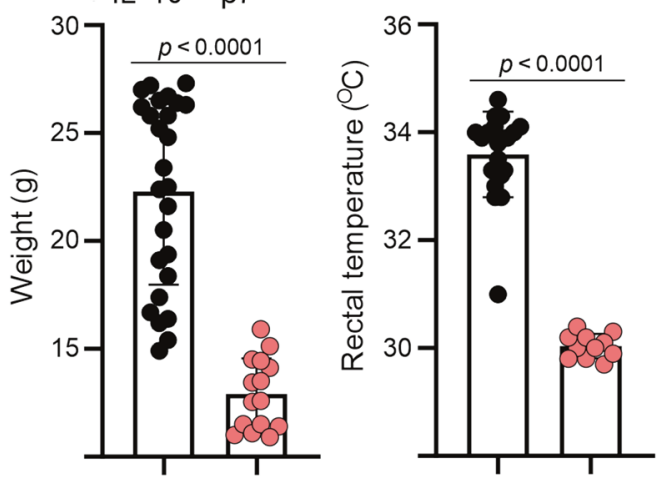

d
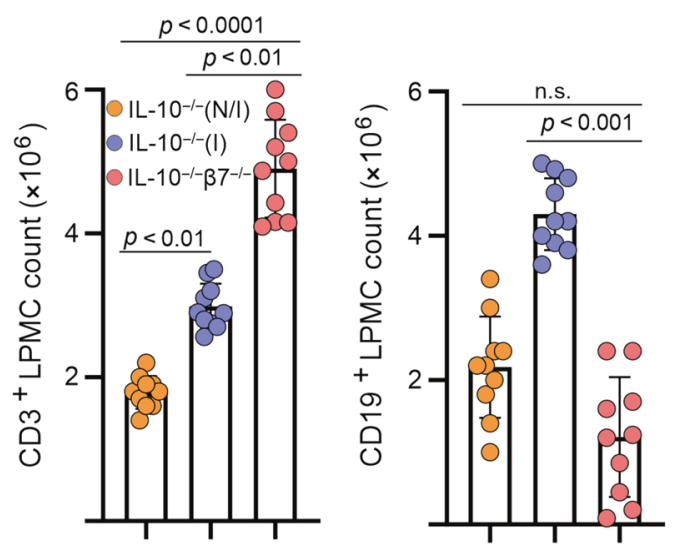

$\mathbf{h}$

IL-10 1 - b

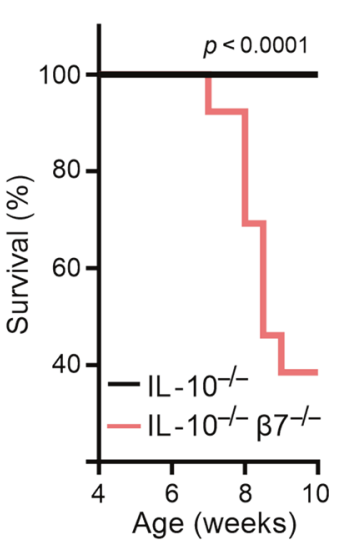

e

f

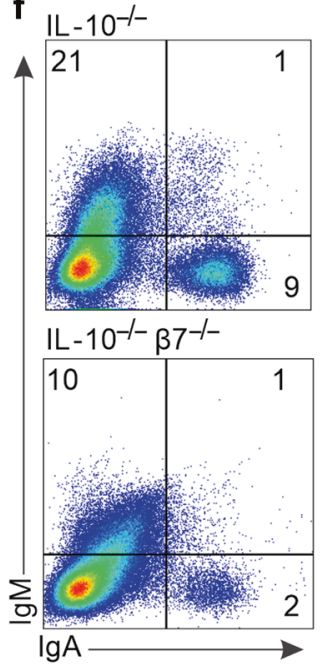

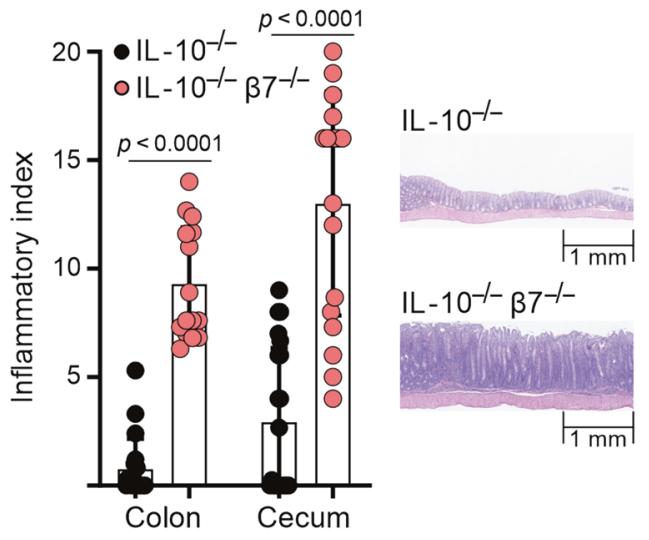

g

OIL-10-/-(N/I)

- IL-10-I-(I)

o|L-10 $0^{-/-} \beta 7^{-/-}$
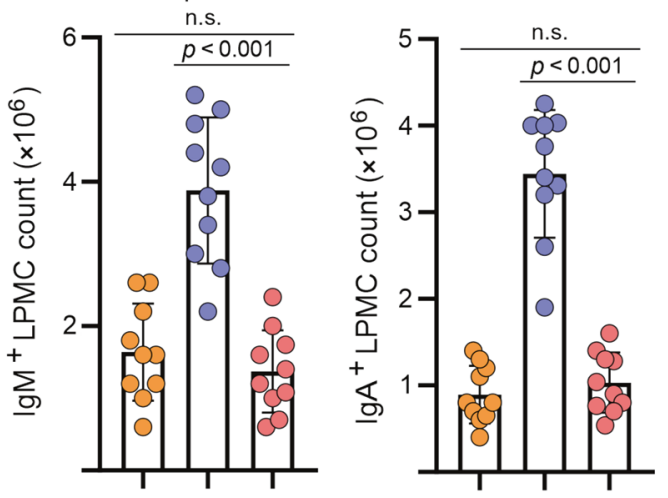
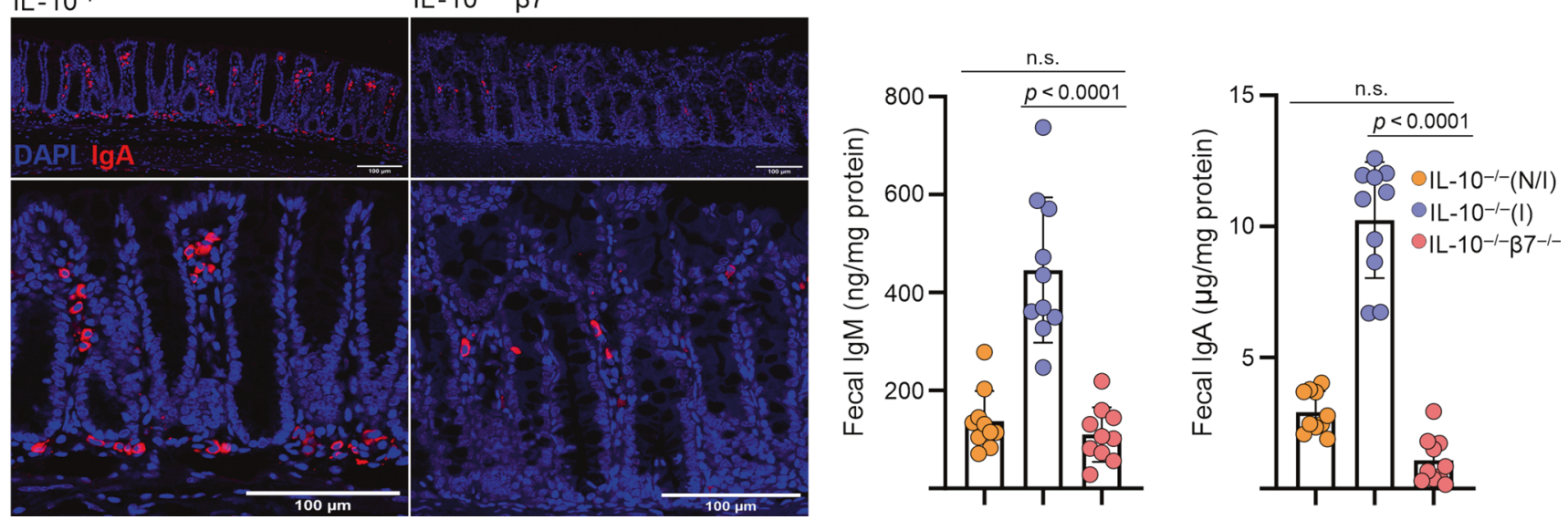

Fig. 2 Integrin $\beta 7$-deficient IL-10 ${ }^{-1-}$ mice display deficits in colonic B cells populations, fecal immunoglobulin levels, and develop more severe colitis. a Weights, rectal temperatures and $\mathbf{b}$ survival curves of indicated strains. $\mathbf{c}$ Colonic and cecal histological indices and representative colonic pathology. $\mathbf{d}$ Colonic LP CD3 ${ }^{+} \mathrm{T}$-cell and e CD19 ${ }^{+}$B-cell counts of indicated strains at 8-12 weeks of age, determined by flow cytometry (noninflamed (N/l, histological index $\leq 1$ ), inflamed (I histological index $>3$ ), freshly isolated LP cells were stained and events gated on live single cells. f Representative flow cytometry plots of colonic LP $\operatorname{lgM}^{+}$and $\operatorname{lgA}{ }^{+}$cells. Events were gated on live, single, $\mathrm{CD}^{-}$, $\mathrm{CD}^{-}$cells. $\mathbf{g}$ Colonic $\mathrm{LP}^{-} \mathrm{IgM}^{+}$and $\mathrm{IgA}^{+}$cell counts of indicated strains. $\mathbf{h}$ Representative confocal immunofluorescence images demonstrating immunolocalization of colonic $\lg \mathrm{A}^{+}$ASC in the indicated mouse strains. i Fecal $\lg \mathrm{M}$ and $\operatorname{lgA}$ of indicated mouse strains. Expression was normalized to total fecal protein. All data are presented as mean \pm S.D, from $n>9$ mice in each dataset. Each data point represents an individual mouse. Statistical significance was determined by ANOVA, followed by Tukey's multiple comparison test (a, $\mathbf{b}$, $\mathbf{d}$, e) or Student's $t$ test $(\mathbf{g}-\mathbf{i})$. 

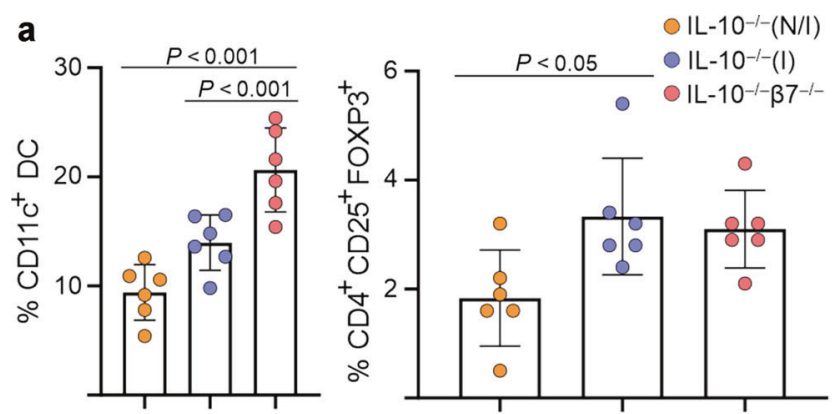

b

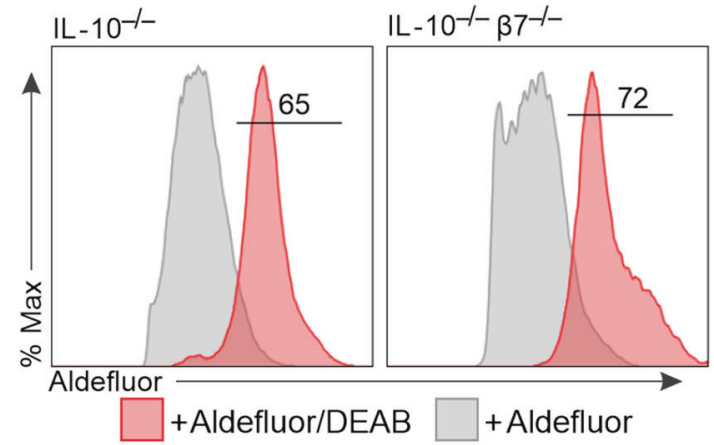

C
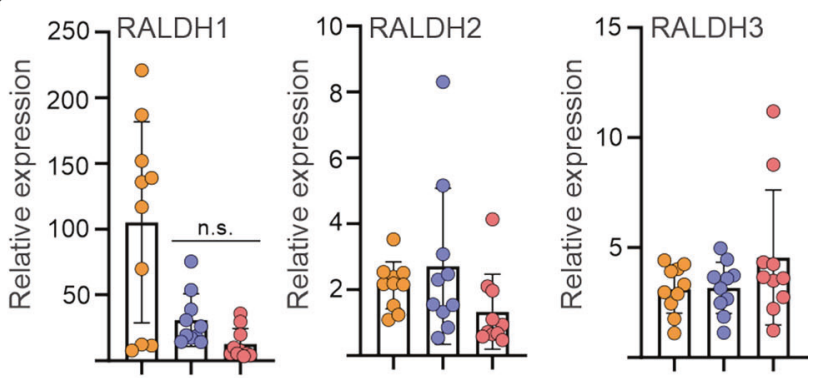

d

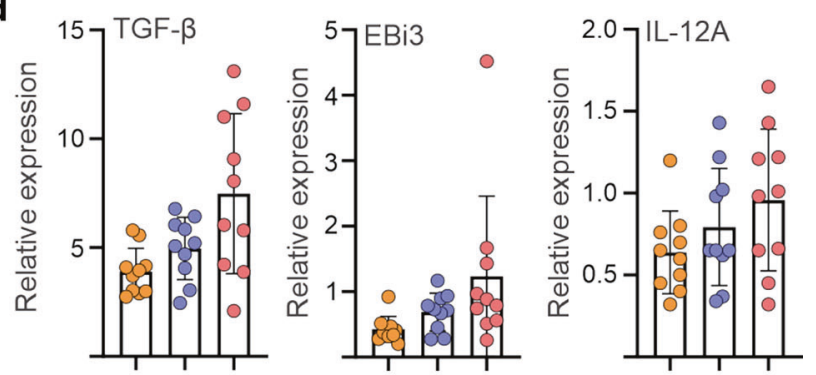

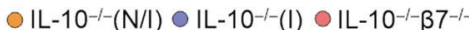

Fig. 3 Dendritic cell and regulatory T-cell numbers, retinaldehyde dehydrogenase activity/mRNA expression, and regulatory B-cellderived cytokines are preserved in $\mathrm{IL}-10^{-1-} / \beta 7^{-1-}$ mice. a Percentage of $\mathrm{CD} 11 \mathrm{c}^{+} \mathrm{DC}$ and $\mathrm{CD}^{+} \mathrm{CD} 25^{+} \mathrm{FOXP}^{+}$regulatory $T$ cells within the colonic LP of the indicated mouse strains, as determined by flow cytometry (noninflamed (N/I), inflamed (I), as determined by histology). Events gated on live, single cells. b Aldefluor conversion within the colonic LP DC fraction isolated from the indicated strains. c Relative mRNA expression of RALDH1, RALDH2, and RALDH3 and $\mathbf{d}$ of regulatory cytokines and subunits within colonic tissues of indicated mouse strains. Expression of the respective genes was normalized to GAPDH expression. Each data point represents an individual mouse. Data are presented as mean \pm S.D from $n>6$ mice in each dataset. Statistical significance determined using ANOVA.
IgA deficiency recapitulates the accelerated phenotype observed in $\beta$ 7-deficient IL-10 ${ }^{-l-}$ mice by inducing bacterial overgrowth and changes in microbiota composition

We then examined the potential association between the $\lg A$ deficit and aggravated colitis stemming from an a4 $\beta 7$-dependent B cell/ ASC recruitment defect and by a congenital IgA deficit. We compared the clinical phenotypes and microbiota composition of $\mathrm{IL}-10^{-/-}, \mathrm{IL}-10^{-/-} / \beta 7^{-/-}$mice, and $\operatorname{lgA}$-deficient (IL-10 $10^{-/-} / \operatorname{lgA}{ }^{-/-}$) mice. Interestingly, IgA-deficient IL-10 ${ }^{-/-}$mice closely paralleled the phenotype of $\mathrm{IL}-10^{-1-} / \beta 7^{-1-}$ mice, inasmuch they developed more severe colitis than IL-10 $0^{-1-}$ mice. This was reflected by lower weights, rectal temperatures, decreased survival (Fig. $5 \mathrm{a}, \mathrm{b}$ ), as well as worse inflammatory indices in both $\mathrm{IL}-10^{-1-} / \beta 7^{-1-}$ and $\mathrm{IL}-10^{-1-} / \mathrm{lgA}^{-1-}$ mice (Fig. 5c). Subsequently, we examined the effects on the microbiota of both the $\beta 7$ and IgA deficits, both resulting in microbial overgrowth and changes in $a$ and $\beta$ diversity (Fig. $5 d-f$ ). Furthermore, although the development of colitis in IL- $10^{-/-}$mice led to baseline taxa redistribution, as compared with noninflamed littermates, both $\beta 7$ and $\lg A$ deficiency resulted in additional changes in taxa, which were clearly distinct from inflamed and noninflamed mice (Fig. 5f). Interestingly, observed changes highly overlapped between $\mathrm{IL}-10^{-/-} / \beta 7^{-/-}$and $\mathrm{IL}-10^{-/-} / \mathrm{lgA}{ }^{-/-}$mice (Fig. $5 \mathrm{f}, \mathrm{g}$ ) primarily represented by decreased Bacteroides and increased Clostridiales. Taken together, these data support a critical ASC dependency on a4 $37 /$ MAdCAM- 1 interactions to migrate to the intestine and maintain luminal SlgA levels required for the maintenance of a homeostatic microbiota.

\section{DISCUSSION}

Understanding the mechanism of action of integrin-based therapeutics by defining their cellular targets may aid in the personalization of treatment options for IBD. We report, herein, that $B$ cells at least in mice are uniquely dependent on $a 4 \beta 7$ to access the colonic LP during chronic inflammation. We demonstrate that $\beta 7$ deficiency and MAdCAM- 1 blockade lead to colonic $B$ cell/ASC deficits, fecal lg deficit, and hastening of colitis, associated with major changes in microbiota composition, distinct from those induced by colitis alone. We also show that both the clinical phenotype and microbiota alterations resulting from defective or blocked a4 $37 / M A d C A M-1$ interactions are recapitulated by $\lg A$ deficiency.

Here, we identify the B-cell lineage as the predominant cell population that expresses a $4 \beta 7$ integrin, both at the periphery and colonic LP of mice. In contrast, other lineages preferentially express $\alpha 4 \beta 1$. It has been proposed that expression of $\alpha 4 \beta 7$ and a4 $\beta 1$ dictates whether immune cells are destined for intestinal or peripheral sites ${ }^{22}$. Although subsets of T, B, NK, and myeloid cells and granulocytes were shown to express both integrins ${ }^{9,10}$, to our knowledge, our study is the first to examine the relative expression of each integrin across cell lineages in parallel, using unbiased high-dimensional techniques. Under homeostatic cell trafficking, a4 $\beta 7$ utilization by $B$ cells represents the main pathway for B-cell extravasation into both GALT and LP ${ }^{9,10,22-24}$. In fact, the consequences of integrin $\beta 7$ and MAdCAM-1 deficiencies are largely restricted to the $B$-cell lineage, including reduced Peyer's patch size and deficits in $B$ cell and $\lg \mathrm{A}^{+}$ASC populations within the $\mathrm{LP}^{4,5}$. On the other hand, B-cell recruitment during chronic intestinal inflammation has been less explored. This is of particular importance because chronic inflammation may modulate integrin/ ligand expression patterns ${ }^{25-29}$, with both $\alpha 4 \beta 7$ and $a 4 \beta 1$ integrins potentially mediating intestinal entry ${ }^{26}$. Such redundancy of inflammatory trafficking pathways may have important implications for the successful targeting of these molecules during IBD. Although our preliminary findings suggest differential expression of the a4 integrins by B cells and other leukocytes, we cannot reach any conclusions on the functional implications of this apparent dichotomy at this time. 
a

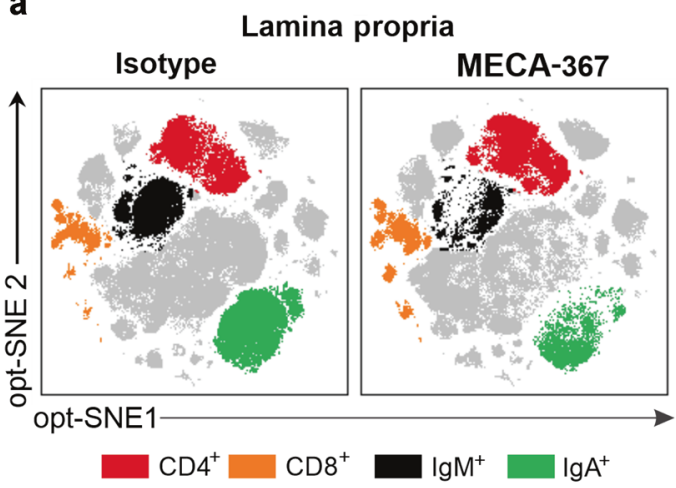

b

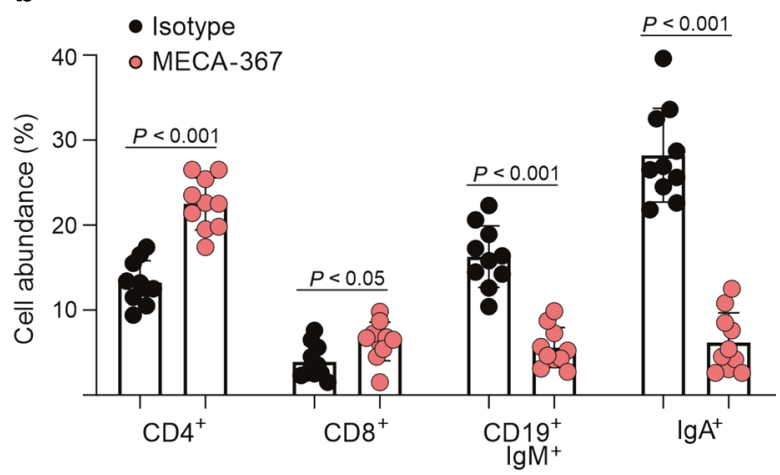

C

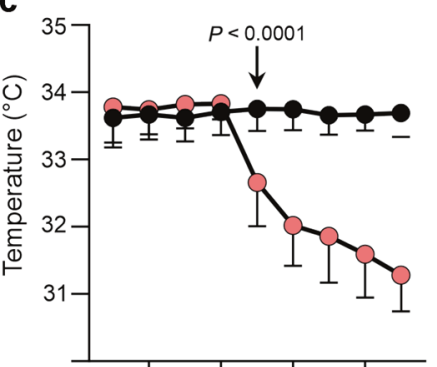

d
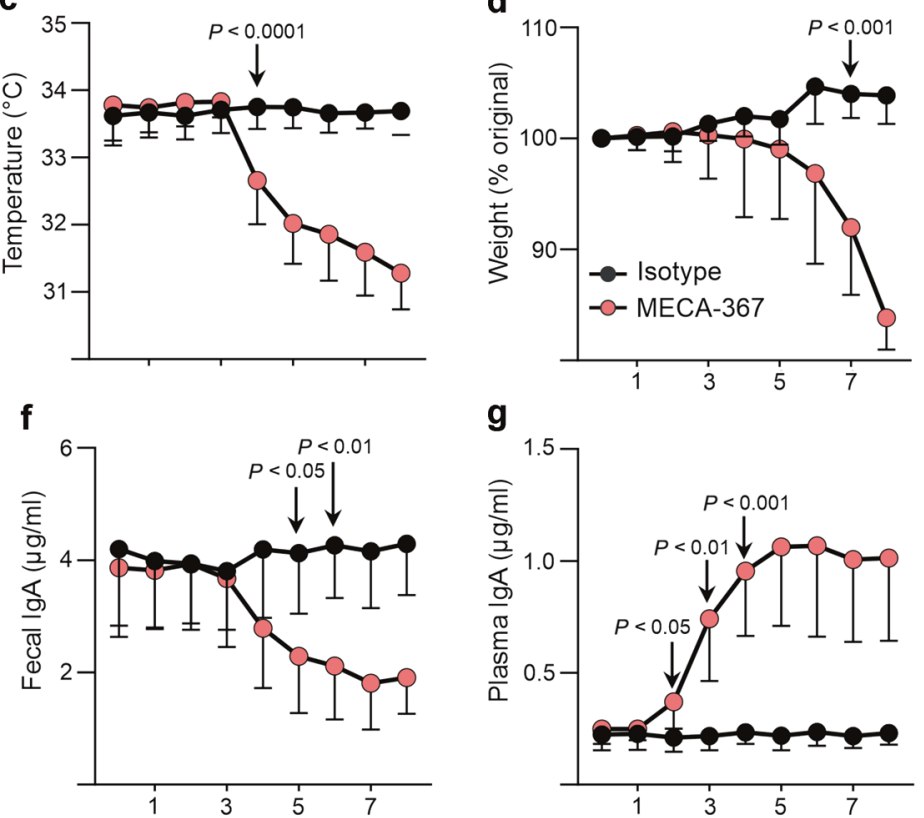

g

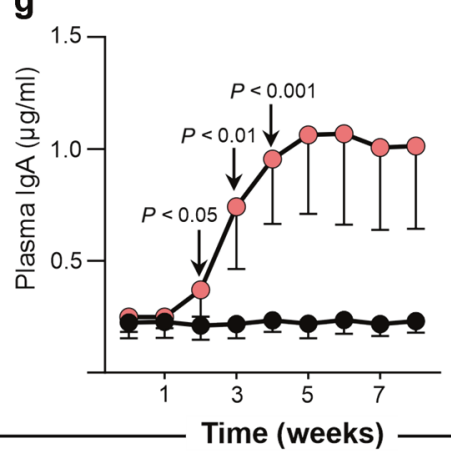

e

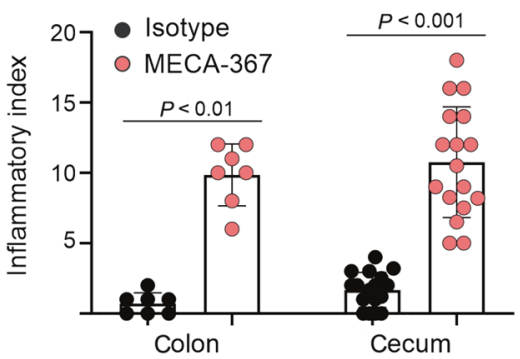

h

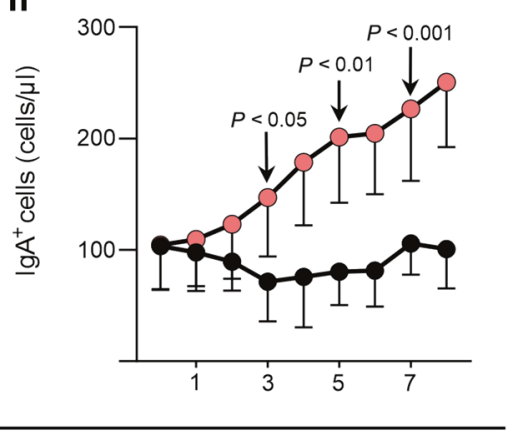

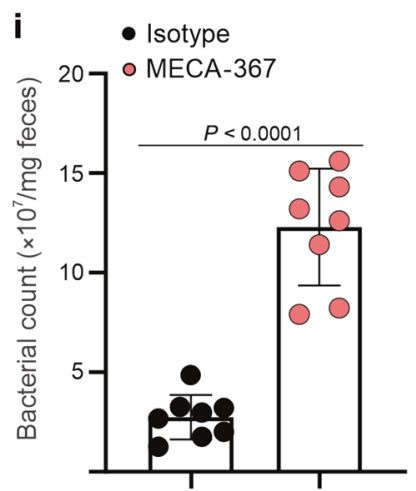

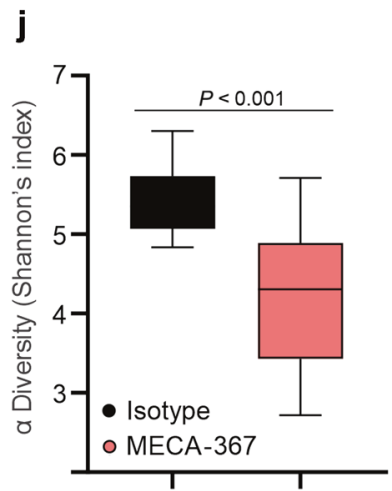

$\mathbf{k}$

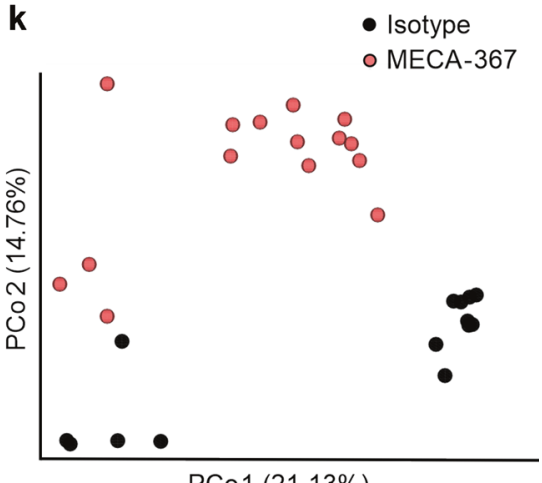

Fig. 4 MAdCAM-1 blockade altered the percentage of B cell/ASC in colon and blood, reversed fecal, and plasma IgA levels, hastened colitis, and induced bacterial overgrowth and changes in microbiota composition. In IL-10 ${ }^{-1-}$ mice, a mass cytometry opt-SNE analysis of concatenated colonic LPMC isolated from mice undergoing the indicated treatments, pregated on nucleated, live, CD45 ${ }^{+}$events. b Percentages of the indicated cell lineages within LPMC fractions of mice treated with MECA-367 or isotype control. c Serial rectal temperatures and $\mathbf{d}$ serial weights. e Colonic and cecal histological indices of treated mice at week 8 . $\mathbf{f}$ Serial fecal lgA and $\mathbf{g}$ plasma lgA levels of treated IL$10^{-/-}$mice. $\mathbf{h}$ Serial peripheral blood cell counts of $\lg \mathrm{A}^{+}$cells. i Fecal bacterial counts from IL-10 ${ }^{-1-}$ mice treated with isotype or MECA-367, determined via RT-qPCR for 16S rRNA expression. $\mathbf{j}$ Alpha diversity (Shannon's index) and $\mathbf{k}$ beta diversity principal coordinate analysis and of $\mathrm{IL}-10^{-1-}$ mice receiving indicated antibodies. All data are presented as mean \pm S.D, from $n>10$ mice in each dataset. Statistical significance was determined by Student's $t$ test (b) or two-way ANOVA, followed by Sidak's multiple comparison test. 
a

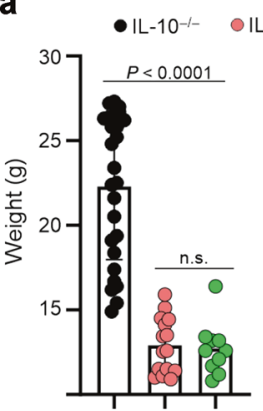

b

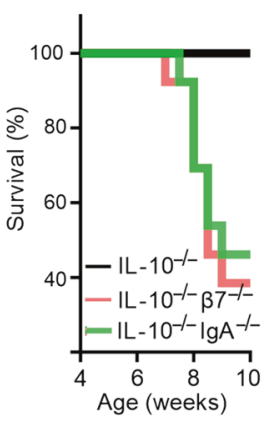

C

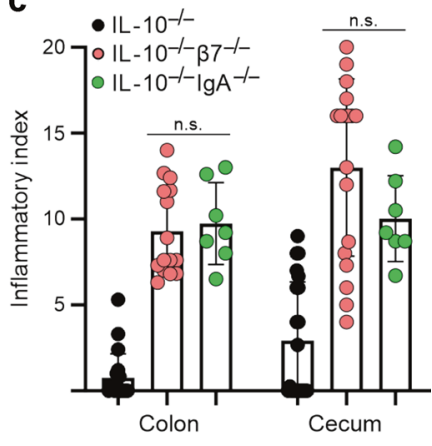

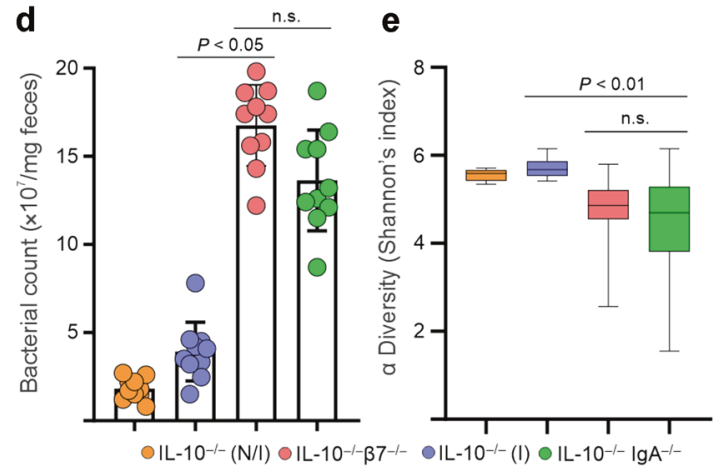

g

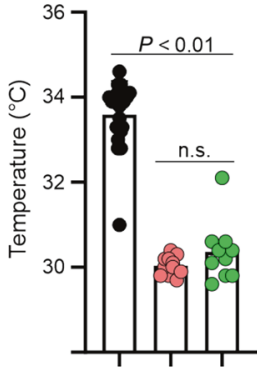

f
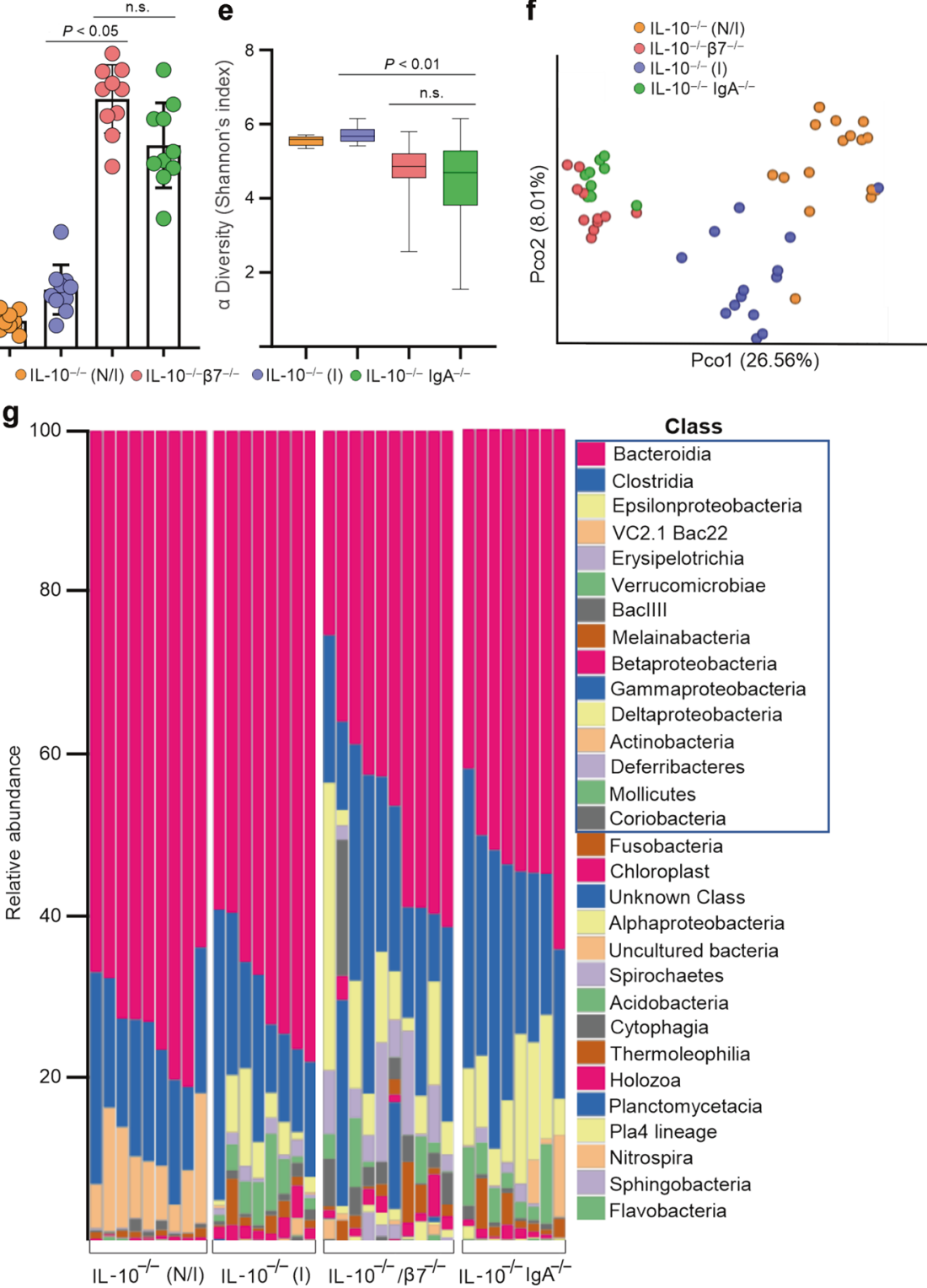

Class

\begin{tabular}{l} 
Bacteroidia \\
Clostridia \\
Epsilonproteobacteria \\
VC2.1 Bac22 \\
Erysipelotrichia \\
Verrucomicrobiae \\
BacllII \\
Melainabacteria \\
Betaproteobacteria \\
Gammaproteobacteria \\
Deltaproteobacteria \\
Actinobacteria \\
Deferribacteres \\
Mollicutes \\
Coriobacteria \\
\hline Fusobacteria \\
Chloroplast \\
Unknown Class \\
Alphaproteobacteria \\
Uncultured bacteria \\
Spirochaetes \\
Acidobacteria \\
Cytophagia \\
Thermoleophilia \\
Holozoa \\
Planctomycetacia \\
Pla4 lineage \\
Nitrospira \\
Sphingobacteria \\
Flavobacteria \\
\end{tabular}

Bacteroidia

Epsilonproteobacteria

VC2.1 Bac22

(acrobiae

Gammaproteobacteria

Deltaproteobacteria

Chloroplast

nknown Class

Alphaproteobacteria

ncultured bacteria

a4 lineage

Sphingobacteria

Fig. 5 Disease phenotype and changes in microbiota observed in $\beta 7$-deficient IL-10 ${ }^{-1-}$ mice are recapitulated by IgA deficiency. a Weights, rectal temperatures, and $\mathbf{b}$ percent survival of indicated strains by 10 weeks of age. $\mathbf{c}$ Colon and cecum histological indices of disease severity of indicated genotypes at 8-12 weeks of age. d Fecal bacterial counts from indicated strains determined via RT-qPCR for 16S rRNA expression. e Fecal bacterial alpha diversity (Shannon's index) and $\mathbf{f}$ beta diversity principal coordinate analysis of the indicated mouse groups. g Relative abundance of bacterial taxa at the class level in feces of the indicated mouse strains. All data are presented as mean \pm S.D, from $n>$ 10 mice in each dataset. Each data point represents an individual mouse. Statistical significance determined using one-way ANOVA, followed by Sidak's multiple comparison test. 
To investigate the role of $\beta 7$ integrins for cell entry into the colonic LP during colitis, we confirmed prior results that showed that $\beta 7$ deficiency leads to significant worsening of colitis in this strain $^{30}$.To understand whether $B$ cells may participate on the immunological basis for such proinflammatory effect of $\beta 7$ deficiency, we examined several different ideas. Firstly, $\beta 7^{-1-}$ mice lack not only $\alpha 4 \beta 7$ but also aE $\beta 7$ integrin. The latter is expressed by intraepithelial lymphocytes and by a subset of intestinal $D C^{19}$ that produce RA, which imprints gut homing and regulatory phenotypes on $T$ lymphocytes and induces $\lg A$ class switching in $\mathrm{ASC}^{2}$. Therefore, aggravated colitis in $\beta 7^{-1-}$ mice could be attributed to the absence of $\mathrm{CD}_{103^{+}} \mathrm{DC}$ and/or defective RA synthesis; nevertheless, we cannot confirm this to be the case as also supported by prior findings that showed that CD103deficient DC are able to induce a gut-homing phenotype ${ }^{31}$, supporting an intact RA enzymatic machinery. On the one hand, no reduction in RA-synthetic enzyme expression, their activity, or Treg progeny was observed in $\beta 7^{-1-}$ mice. Furthermore, an antiMAdCAM-1 antibody, which does not bind CD103, also hastened colitis in adult $\mathrm{IL}-10^{-1-}$ mice. Secondly, a compromise in regulatory mechanisms may also explain the worsening of colitis. Indeed a role of $\alpha 4 \beta 7$ for intestinal recruitment of Tregs has been proposed, and Treg blockade leads to worse colitis in IL-10 ${ }^{-1-}$ mice $^{30}$. However, under our unique experimental conditions, we neither observed a Treg or Breg deficit nor deficit of their regulatory cytokines in either IL-10 ${ }^{-/-} / \beta 7^{-1-}$ or MECA-367 treated mice $^{20}$.

Our findings allow us to propose an alternate or concurrent explanation for the aggravation of colitis in $\beta 7$-deficient IL- $10^{-1-}$ mice via intestinal dysbiosis due to a deficient ASC recruitment from PP leading to an intraluminal deficit of $\lg A$ and other immunoglobulins. These effects were also observed after antiMAdCAM- 1 treatment, and in IgA-deficient IL- $10^{-1-}$ mice, pointing to a common downstream pathway of luminal SlgA deficit leading to dysbiosis. Our findings are in line with a critical role attributed to SIgA for the maintenance of a homeostatic microbiota ${ }^{21}$. Indeed, Fagarasan et al. showed a dramatic expansion of Clostridia in mice that lack activation-induced cytidine deaminase, an essential enzyme for class switch recombination and somatic hypermutation $^{32}$. A similar expansion of Clostridia species was also detected in our study and recapitulated in IgA-deficient IL-10 ${ }^{-1-}$ mice. Recently, minimal distortion of the resident microbiota composition was found in $\beta 7^{-1-}$ mice under homeostatic conditions, despite significant perturbation of the intestinal B-cell compartment ${ }^{33}$. However, it is possible that a baseline dysregulation of the microflora that is known to occur in inflamed IL- $10^{-1-}$ mice $^{34}$ is required for the accentuated effect of $\beta 7 / \lg A$ deficiency to take place. Nevertheless, whether dysbiosis or inflammation is the primary event remains to be determined. The effect of MAdCAM-1 blockade on the microbiota and colitis severity in IL-10 $10^{-1-}$ cohoused siblings supports a causal effect, induced by the resultant luminal SIgA deficit, expansion of a dysbiotic flora, barrier breach, and possible systemic sepsis as reflected by hypothermia. In contrast, we observed broad distortion of microbiota composition following onset of colitis in $\beta 7-$ deficient IL- $10^{-1-}$ mice, which may be reflective of impaired $B$-cell recruitment and inability to meet the demand for increased luminal SIgA, brought on by inflammation.

The increased severity of colitis in $\beta 7$-deficient $\mathrm{IL}-10^{-/-}$mice shown herein is in contrast with the majority of published studies in mouse models of colitis, which report anti-inflammatory effects of $\beta 7$ deficit or anti-MAdCAM-1 antibody blockade ${ }^{35}$. In fact, the only exceptions so far have been the aggravated/lethal colitides that take place in $\beta$-deficient IL-10 ${ }^{-/-}$mice, a B-cell deficit ( $\mu \mathrm{MT} /$ IL-10 ${ }^{-1-}$ cross) ${ }^{36}$, MAdCAM-1 blockade ${ }^{30}$, and in colitis-prone Gai2 mice after treatment with anti-a4 integrin antibody ${ }^{37}$. Those models are methodologically distinct from all others because the treatment course extended beyond 6 weeks. The timeline might be critical, because long-lived plasma cells are able to maintain fecal IgA levels for weeks, before the compromised plasmablast recruitment finally tilts the balance ${ }^{38}$. Of note, in our MAdCAM-1 blockade studies increases in serum $\lg A$ and peripheral B cell/ASC counts predated fecal IgA deficit by 2 weeks, suggesting that these cells accumulate in peripheral blood when unable to reach the intestine. Clinically, hypothermia also predated weight loss by 2 weeks, suggesting that the inflammatory process and potential barrier breach begins much earlier that it is manifested clinically by the uniformly used weight loss parameter. The MAdCAM-1 blockade model shows a dynamic process whereby effects on plasmablast recruitment result in converse effects in peripheral blood and LP, eventually leading to hypothermia, weight loss, and aggravated colitis. The timeline of integrin blockade may have implications for the mechanism of slow acting ${ }^{39}$ integrin-based therapies, due to potential differential effects on short-lived and long-lived cell subsets, the latter being less dependent on integrin-based recruitment for replenishment ${ }^{6,7}$. Interestingly, plasma and fecal IgA levels plateaued only after going through several weeks of increases/decreases, respectively, indicating that stabilization of the number of $\mathrm{LP}_{\operatorname{lgA}}{ }^{+} \mathrm{ASC}$ may take time. This may be explained by the existence of a subpopulation of $B$ cells, which utilize alternative integrins for intestinal entry (e.g., a4 $\beta 1$ ), or may be due to long-lived plasma cells ${ }^{38}$ which may survive past the 8-week treatment protocol. The phenotype, integrin expression profile, and lifespan of $\lg \mathrm{A}^{+} \mathrm{ASC}$ that persist after sustained integrin/ligand blockade remains to be investigated.

Our study has potential translational implications for the treatment of patients with IBD. Firstly, it emphasizes the pathogenetic association and therapeutic potential of $B$ cells in IBD, which have been largely underappreciated. This could be in part due to the failure of rituximab (anti-CD20 antibody) in $U^{40}$. Although it is known that CD20 is only expressed by a subset of cells of the B lineage. Similarly, most animal studies that examined the effects of trafficking blockade have focused almost exclusively on the effects on T cells, whereas the impact of the B cell/ASC/ SIgA deficits has not been considered, or even dismissed as irrelevant $^{36}$. Secondly, our study clearly highlights B cells as the principal a4 $\beta 7$-expressing population, uniquely dependent on a4ß7:MAdCAM-1 for intestinal trafficking under inflammatory conditions. Human $\mathrm{B}$ cells also bind $\mathrm{VDZ}^{10}$ and the drug decreases $B$ cells and lymphoid aggregates (inducible lymphoid follicles) in the intestine of HIV-1 infected individuals ${ }^{41}$. It also modulates the B-cell compartment in IBD ${ }^{12,42}$ and inhibits antibody responses to oral vaccination, even after a single dose ${ }^{43}$. In contrast, $T$ cells express $\alpha 4 \beta 7$ and $\alpha 4 \beta 1$ heterogeneously ${ }^{44}$, whereas NK cells bind MAdCAM-1 poorly ${ }^{22}$. Those differential expression patterns of a $4 \beta 7$ and $a 4 \beta 1$ integrins may have important implications for the clinical efficacy of drugs that target trafficking pathways. Currently, such therapies target either both a4 integrins (i.e., natalizumab), or $\beta 7$ integrins (i.e., VDZ, etrolizumab), or MAdCAM-1 (i.e., ontamalimab). Thirdly, we describe the accumulation of B-cell subsets and Ig in blood after MAdCAM-1 blockade, similarly to splenic accumulation of $\lg \mathrm{A}^{+}$cells in $\beta 7^{-1-}$ mice ${ }^{45}$. Peripheral accumulation of $\alpha 4 \beta 7^{+} \mathrm{CD}^{+} \mathrm{T}$ cells after VDZ treatment ${ }^{26,46}$ and MAdCAM1 blockade $^{47}$ has been reported previously. Those changes in peripheral cell frequency and serum immunoglobulin concentrations hold promise for the development of clinically useful biomarkers of response to anti-integrin therapies.

It remains difficult to reconcile the therapeutic effect of a4 $\beta 7$ MAdCAM-1 blockade seen in patients treated with VDZ or ontamalimab (anti-MAdCAM-1) with the hastening effect of a parallel intervention on IL-10 ${ }^{-1-}$ colitis. First, evidence continues to accumulate that our understanding of the mode of action of VDZ and other anti-integrin therapies is incomplete. Second, the discrepancy could be attributed to a limited translational value of this specific model to human UC. However, a similar result was also reported in Gai colitic mice ${ }^{37}$. Discrepancy with other 
preclinical immunoblockade studies could be additionally explained by the duration of treatment (less than 4 weeks in most studies) ${ }^{35}$. Of note is that we do not see a reduction in fecal IgA until week 5 of MAdCAM-1 blockade, as luminal SIgA is likely maintained by long-lived plasma cells that are less dependent on replenishment by IgA plasmablasts from blood. Alternatively, our preclinical observations might be more reflective of human IBD at its earliest stages (disease onset), triggered by loss of tolerance to bacterial antigens. This early stage of IBD is subclinical in humans and likely distinct from established clinical IBD, once the dysregulated immune process has become self-sustaining and less dependent on continuous bacterial antigenic stimulation. It is during this maintenance stage when patients present for medical care and are treated with VDZ, likely years after the onset of the initial dysregulated immune process.

Finally, while our data identify B cells as uniquely dependent on a4 $\beta 7$ for colonic entry in mice, it is clear that a4 $\beta 7$ is expressed and may be important for intestinal homing of other immune cell lineages in mice and humans. Schleier and colleagues reported that $\alpha 4 \beta 7$ is expressed by nonclassical monocytes, as defined by CD14 and CD16 coexpression (CD14+ $\mathrm{CD} 16++)$ and that these cells increase in peripheral blood of VDZ-treated patients ${ }^{13}$. Indeed, VDZ modulates innate cell populations, importantly macrophages (increased M1, decreased M2). In another study however, adhesion of $\mathrm{CD}^{+}$ $\mathrm{T}$ cells to MAdCAM-1 predicted responsiveness to VDZ in $\mathrm{UC}^{46}$. Thus, much remains to be learned regarding the definite mode of action of these drugs.

In conclusion, our data reiterate the critical dependence of $B$ cells/ASC on a4 $37 / M A d C A M-1$ interactions for intestinal recruitment, IgA production, and maintenance of a homeostatic microbiota. We propose that during chronic colitis in mice, most $B$ cells are uniquely reliant on a4 $\beta 7$ pathways for intestinal recruitment, being unable to circumvent $a 4 \beta 7$ deficiency, perhaps via a4 $\beta 1$-VCAM-1 interactions or other integrin pathways. This may have important implications for personalization of antiintegrin therapies as antibodies or small molecules that target both a4 integrins may be more efficacious in certain patient subsets (e.g., Crohn's vs. UC). The relative dependence of different cell lineages on distinct integrins may define efficacy of a particular anti-integrin drug on individual patients.

\section{MATERIALS AND METHODS}

Mice

$\mathrm{IL}-10^{-/-}$and integrin $\beta 7^{-/-}$mice of the C57BL/6 background were obtained from The Jackson Laboratory (Stock nos.: 002251 and 002965 , respectively) and bred in-house. IgA ${ }^{-/-}$mice of the same background were a kind gift from Lars Eckmann ${ }^{48}$. Mice were cross-bred to homozygous state of the relevant gene mutations and genotype was confirmed by Transnetyx Inc (TN, USA).

For MAdCAM-1 and a4 blockade experiments, cohorts of 8-week-old IL$10^{-1-}$ mice were treated with either $100 \mu \mathrm{g}$ of MECA-367 (anti-MAdCAM1), PS-2 (anti-integrin a4), or rat lgG2a isotype control (both BioxCell, $\mathrm{NH}$, USA), twice weekly, for a period of 8 weeks. Weekly weights, rectal temperatures, plasma, and fecal samples were collected throughout the course of the experiment. Cohorts of mice were euthanized and tissues harvested once MECA-367-treated mice had lost $20 \%$ of their original weight.

For mouse survival curves, mice were euthanized once reached $80 \%$ original body weight and this timepoint was recorded as death. Mouse colonic and cecal tissues were prepared for histological analysis by fixation in 10\% neutral buffered formalin (Thermo Fisher Scientific, MA, USA), followed by $70 \%$ ethanol (VWR, PA, USA), embedded in paraffin, and cut into vertical $4-\mu m$-thick sections. Tissue sections were stained with hematoxylin and eosin and histological assessment of inflammation was performed using a standardized semiquantitative scoring system ${ }^{18}$, by a trained pathologist (P.J.) blinded to the experimental conditions. Murine blood was obtained via submandibular bleed during the course of experiments or via cardiac puncture bleed at the time of tissue harvest. Serum was separated and stored at $-80^{\circ} \mathrm{C}$.

\section{Cell isolation}

Splenocytes were isolated via mechanical disruption of whole spleen, followed by red blood cell (RBC) lysis (RBC lysis buffer, BioLegend). Leukocytes were obtained from whole blood via two rounds of RBC lysis. Colonic LP cells were obtained as previously described ${ }^{18}$. In brief, colonic tissues were washed for $15 \mathrm{~min}$ at room temperature in $15 \mathrm{~mL}$ HBSS (Fisher Scientific), followed by three washes in HBSS + 1 mM EDTA (Hoefer Inc, MA, USA), followed by one wash in HBSS. Tissues were then subject to mechanical disruption and digestion for $45 \mathrm{~min}$, in a solution containing complete RPMI $+1.5 \mathrm{mg} / \mathrm{mL}$ collagenase VIII and $1 \mu \mathrm{M}$ DNase (both Sigma Aldrich). Resulting cell suspensions were filtered and utilized for downstream analyses.

\section{Mass cytometry antibody staining and barcoding}

Cell suspensions were prepared for mass cytometry acquisition as previously described ${ }^{49}$, but with additional barcoding steps. In brief, isolated cells were resuspended in Maxpar cell staining buffer [CSB; Fluidigm, San Francisco, CA] and subsequently stained as follows: (1) viability staining using Cisplatin-195Pt [Fluidigm] for $5 \mathrm{~min}$; (2) initial fixation step using Maxpar Fix I buffer [Fluidigm] for 20 min; (3) permeabilization and barcode labeling of each sample using the Cell-ID 20-Plex Pd Barcoding Kit [Fluidigm]. Following barcoding, cells from up to 20 different samples were combined into a single tube for further antibody labeling; (4) Fc receptor blockade using Mouse TruStain FcX [BioLegend, San Diego, CA] for $10 \mathrm{~min}$; (5) surface antibody staining [Fluidigm; BioLegend, San Diego, CA, full antibody panels in Supplementary Table 1] for $30 \mathrm{~min}$; (6) second fixation step using $1.6 \%$ formaldehyde [Methanolfree, Thermo Scientific] for $10 \mathrm{~min}$; and (7) DNA-intercalator labeling using Maxpar Fix \& Perm Buffer [Fluidigm] and Cell-ID Intercalator-Ir [Fluidigm], incubated at $4{ }^{\circ} \mathrm{C}$ overnight. Following overnight intercalator staining, samples were washed twice with CSB and stored at $-80^{\circ} \mathrm{C}$, in $90 \% \mathrm{FBS}$, $10 \% \mathrm{DMSO}^{49}$. Where indicated, purified antibodies were conjugated with metal isotopes in-house, using antibody labeling kits [Fluidigm]. For barcoding distribution of samples, equal numbers of samples from each sample group were distributed across multiple barcode sets, to ensure comparable data acquisition and to minimize batch effects in the downstream data analysis.

\section{Mass cytometry sample acquisition and data processing}

Prior to acquisition, cells were washed twice with Milli-Q water and resuspended in a 1:10 dilution of EQ Four Element Calibration Beads [Fluidigm] to a concentration of $0.5 \times 10^{6}$ cells $/ \mathrm{mL}$. Samples were acquired using a CyTOF Helios [Fluidigm] and data normalized to mass bead signal using the Nolan lab Matlab software [GitHub, https://github.com/nolanlab]. Each Barcode set was fully acquired within 1 day of mass cytometry run time, and all barcode sets were acquired on sequential days.

\section{Mass cytometry data analysis}

Following normalization, barcoded samples were debarcoded using the Nolan lab single-cell debarcoder tool [GitHub, https://github.com/ nolanlab]. Mass cytometry data were analyzed using OMIQ [OMIQ, Inc] for biaxial gating, opt-SNE, FlowSOM, and edge $\mathrm{R}^{50}$ analytical algorithms. Prior to t-distributed stochastic neighbor embedding [t-SNE] analysis, data were gated on nucleated, live, $\mathrm{CD} 45^{+}$events, then gated on the indicated populations of interest. Unless otherwise stated, opt-SNE analyses were conducted using 100,000 total events proportionally drawn from samples, with 1000 iterations and a perplexity value of 30 . Where indicated, opt-SNE plots displayed were produced by concatenating multiple files into a single image, post analysis. FlowSOM analyses were conducted following opt-SNE analysis, using the Euclidean distance metric and consensus metaclustering with a comma-separated $k$ value of 25 . EdgeR analysis was performed on all identified clusters for each lineage described, and $P$ values below 0.01 were utilized to determine significant differences. Summary graphs of cell abundances were produced using GraphPad Prism version 8 software [GraphPad Software Inc, La Jolla, CA].

\section{Flow cytometry}

Viability staining was conducted using fixable aqua dead cell stain [Thermo Fisher Scientific] according to the manufacturer's instructions. Fc receptor blockade was performed using TruStain FCX anti-mouse CD16/32 [both BioLegend]. Extracellular antibody staining was performed using the following antibodies: CD3 BV785, CD4 FITC, CD8 PE/Cy5, CD19 BV711, CD25 PE, integrin 37 BB515, CD49d PerCP/Cy5.5, CD11b BV605, CD11C 
BV711, IgA PE, IgM APC efluor 780, IgG BV421, and MHCII PerCP/Cy5.5. Following staining, cells were fixed using the $B D$ stabilizing fixative solution [BD Biosciences]. Where required, intracellular staining was performed using the FOXP3 Fix/Perm Buffer Set (BioLegend), according to the manufacturer's instructions, as well as anti-mouse FOXP3 BV421 (BioLegend). Cells were acquired on a 16-color BD LSRII [BD Biosciences]. Where indicated, cells were acquired alongside AccuCheck counting beads [Thermo Fisher Scientific] for absolute cell counts. Where applicable, cells were incubated with Aldefluor reagent (Stemcell Technologies, Vancouver, $\mathrm{BC}$, Canada) to identify cells that express high levels of aldehyde dehydrogenase (ALDH). The activated reagent, BODIPY-aminoacetaldehyde, is a fluorescent substrate for ALDH that diffuses into viable cells and is converted to BODIPY-aminoacetate and retained intracellularly. The fluorescent reaction product is proportional to the ALDH activity. Diethylaminobenzaldehyde is a specific inhibitor of ALDH used to control for background fluorescence. Flow cytometry data were analyzed using FlowJo version 10 (Treestar, OR, USA).

\section{Soluble immunoglobulin measurements}

Fecal and plasma murine immunoglobulin levels were determined using the Mouse Isotyping Panel 1 Kit (Meso Scale Discovery, MD, USA). Fecal pellets were vortexed in $1 \mathrm{~mL}$ of PBS for $10 \mathrm{~min}$, centrifuged at $12000 \times \mathrm{g}$ for $10 \mathrm{~min}$ twice, and supernatant collected for Ig level determination. Plasma was diluted at 1:10,000 and fecal supernatant was diluted at 1:100 for analysis. Fecal Ig levels were normalized per mg of total fecal protein content, as determined by Bradford Protein Assay (Bio-Rad, CA, USA). Plates were read using a MESO QuickPlex SQ 120, and analyzed using the MSD workbench software.

\section{Immunofluorescence}

Four-micrometer-thick paraffin sections were deparaffinized, rehydrated, and permeabilized with Triton X-100 $0.01 \%$ in PBS for $1 \mathrm{~h}$ at room temperature, blocked with $1 \%$ BSA in PBS for $1 \mathrm{~h}$, then stained with $2.5 \mu \mathrm{g} /$ $\mathrm{mL}$ anti-mouse IgA primary antibody (556969; BD Pharmingen, NJ, USA) in blocking buffer ( $1 \%$ BSA in PBS) for $18 \mathrm{~h}$ at $4^{\circ} \mathrm{C}$. Sections were subsequently incubated with $2 \mu \mathrm{g} / \mathrm{mL}$ Alexa Fluor 594 goat anti-rat lgG $(\mathrm{H}+\mathrm{L})$ secondary antibody (A-11007, Thermo Fisher Scientific, MA, USA) for $2 \mathrm{~h}$ at $4{ }^{\circ} \mathrm{C}$. After washing, specimens were mounted with ProLong ${ }^{\mathrm{TM}}$ Gold Antifade Mount with DAPI (P36935, Thermo Fisher Scientific, MA, USA) and stored at $4{ }^{\circ} \mathrm{C}$. Image acquisition was performed with a Zeiss LSM780 confocal microscope (Zeiss, Jena, Germany) equipped with a $40 \times /$ 1.3 NA EC Plan-Neofluar oil objective. High-resolution images of the whole sample were taken through a tile scan.

\section{Microbiota analysis}

Fecal samples were stored at $-80^{\circ} \mathrm{C}$ until use. DNA was extracted using the QIAamp DNA Stool Mini Kit (Qiagen, Germany), according to the manufacturer's guidelines. V4 fragments of the 16S rRNA gene were sequenced using an Illumina MiSeq platform. Sequencing datasets were analyzed using the QIITA online analysis platform. Sequencing files were demultiplexed, trimmed to 150 base pairs, deblurred, and operational taxonomic unit assigned using the Silva-119 database. Data were rarified, alpha diversity determined using Shannon's index, and beta diversity determined using the Bray-Curtis dissimilarity, followed by principal coordinate analysis.

\section{Real-time qPCR}

Colonic tissues were harvested and stored at $-80^{\circ} \mathrm{C}$ in RNAlater solution (Thermo Fisher Scientific). RNA was isolated from colonic tissues using the RNeasy Mini Kit (Qiagen, Germany). cDNA was generated using the High Capacity cDNA Reverse Transcription Kit (Applied Biosystems, CA, USA). Gene expression was examined using the following Taqman probes (all Thermo Fisher Scientific): TGF $\beta$ (Tgif1, Mm01227699_m1), EBI3 (Mm00469294_m1), IL-12A (Mm00434169_m1), RALDH1 (Aldh1a1, Mm00657317_m1), RALDH2 (Aldh1a2, Mm00501306_m1), and RALDH3 (Aldh1a3, Mm00474049 m1), and normalized to GAPDH (Mm99999915_g1).

\section{Statistics}

Statistical analysis was performed using GraphPad Prism 8 software [GraphPad Software Inc]. For comparisons of two groups, the Student's $t$ test was utilized. For comparison of multiple groups, ANOVA was utilized, followed by Tukey's multiple comparison test. Descriptive statistics are displayed as mean \pm standard deviation in all figures. Significance is defined as $P$ values of $<0.05$, resulting statistical significances of difference are indicated in figures as $P<0.05, P<0.01, P<0.001$, and $P<0.0001$, and n.s represents comparisons which are not statistically significant in difference.

\section{REFERENCES}

1. Brandtzaeg, P., Carlsen, H. S. \& Halstensen, T. S. The B-cell system in inflammatory bowel disease. Adv. Exp. Med Biol. 579, 149-167 (2006).

2. Mora, J. R. et al. Generation of gut-homing IgA-secreting B cells by intestinal dendritic cells. Science 314, 1157-1160 (2006).

3. von Andrian, U. H. \& Mackay, C. R. T-cell function and migration. Two sides of the same coin. N. Engl. J. Med. 343, 1020-1034 (2000).

4. Schippers, A. et al. Mucosal addressin cell-adhesion molecule-1 controls plasmacell migration and function in the small intestine of mice. Gastroenterology 137, 924-933 (2009).

5. Wagner, N. et al. Critical role for beta7 integrins in formation of the gutassociated lymphoid tissue. Nature 382, 366-370 (1996).

6. Feagan, B. G. et al. Vedolizumab as induction and maintenance therapy for ulcerative colitis. N. Engl. J. Med. 369, 699-710 (2013).

7. Sandborn, W. J. et al. Vedolizumab as induction and maintenance therapy for Crohn's disease. N. Engl. J. Med. 369, 711-721 (2013).

8. Vermeire, S. et al. Etrolizumab as induction therapy for ulcerative colitis: a randomised, controlled, phase 2 trial. Lancet 384, 309-318 (2014).

9. Erle, D. J. et al. Expression and function of the MAdCAM-1 receptor, integrin alpha 4 beta 7, on human leukocytes. J. Immunol. 153, 517-528 (1994).

10. Soler, D. et al. The binding specificity and selective antagonism of vedolizumab, an anti-alpha4beta7 integrin therapeutic antibody in development for inflammatory bowel diseases. J. Pharm. Exp. Ther. 330, 864-875 (2009).

11. Rogler, G. Mechanism of action of vedolizumab: do we really understand it? Gut 68, 4-5 (2019).

12. Zeissig, S. et al. Vedolizumab is associated with changes in innate rather than adaptive immunity in patients with inflammatory bowel disease. Gut 68, 25-39 (2019).

13. Schleier, L. et al. Non-classical monocyte homing to the gut via alpha4beta7 integrin mediates macrophage-dependent intestinal wound healing. Gut 69, 252-263 (2020).

14. Noor, N. M., Verstockt, B., Parkes, M. \& Lee, J. C. Personalised medicine in Crohn's disease. Lancet Gastroenterol. Hepatol. 5, 80-92 (2020).

15. Keubler, L. M., Buettner, M., Hager, C. \& Bleich, A. A multihit model: colitis lessons from the interleukin-10-deficient mouse. Inflamm. Bowel Dis. 21, 1967-1975 (2015).

16. Kullberg, M. C. et al. Helicobacter hepaticus triggers colitis in specific-pathogenfree interleukin-10 (IL-10)-deficient mice through an IL-12- and gamma interferon-dependent mechanism. Infect. Immun. 66, 5157-5166 (1998).

17. Zundler, S. et al. The alpha4beta1 homing pathway is essential for ileal homing of Crohn's disease effector t cells in vivo. Inflamm. Bowel Dis. 23, 379-391 (2017).

18. Burns, R. C. et al. Antibody blockade of ICAM-1 and VCAM-1 ameliorates inflammation in the SAMP-1/Yit adoptive transfer model of Crohn's disease in mice. Gastroenterology 121, 1428-1436 (2001).

19. Kilshaw, P. J. Expression of the mucosal T cell integrin alpha M290 beta 7 by a major subpopulation of dendritic cells in mice. Eur. J. Immunol. 23, 3365-3368 (1993).

20. Mizoguchi, A., Mizoguchi, E., Smith, R. N., Preffer, F. I. \& Bhan, A. K. Suppressive role of $B$ cells in chronic colitis of T cell receptor alpha mutant mice. J. Exp. Med. 186, 1749-1756 (1997).

21. Brandtzaeg, P. Secretory IgA: designed for anti-microbial defense. Front. Immunol. 4, 222 (2013).

22. Rott, L. S., Briskin, M. J., Andrew, D. P., Berg, E. L. \& Butcher, E. C. A fundamental subdivision of circulating lymphocytes defined by adhesion to mucosal addressin cell adhesion molecule-1. Comparison with vascular cell adhesion molecule-1 and correlation with beta 7 integrins and memory differentiation. J. Immunol. 156, 3727-3736 (1996).

23. Rott, L. S., Briskin, M. J. \& Butcher, E. C. Expression of alpha4beta7 and E-selectin ligand by circulating memory $B$ cells: implications for targeted trafficking to mucosal and systemic sites. J. Leukoc. Biol. 68, 807-814 (2000).

24. Farstad, I. N. et al. Human intestinal B-cell blasts and plasma cells express the mucosal homing receptor integrin alpha 4 beta 7. Scand. J. Immunol. 42, 662-672 (1995).

25. Wittner, M. et al. Comparison of the integrin alpha4beta7 expression pattern of memory $T$ cell subsets in HIV infection and ulcerative colitis. PLOS ONE 14, e0220008 (2019). 
26. Fuchs, F. et al. Clinical response to vedolizumab in ulcerative colitis patients is associated with changes in integrin expression profiles. Front. Immunol. 8, 764 (2017).

27. Briskin, M. et al. Human mucosal addressin cell adhesion molecule- 1 is preferentially expressed in intestinal tract and associated lymphoid tissue. Am. J. Pathol. 151, 97-110 (1997).

28. Jones, S. C. et al. Adhesion molecules in inflammatory bowel disease. Gut 36, 724-730 (1995)

29. Gu, P., Theiss, A., Han, J. \& Feagins, L. A. Increased cell adhesion molecules, PECAM-1, ICAM-3, or VCAM-1, predict increased risk for flare in patients with quiescent inflammatory bowel disease. J. Clin. Gastroenterol. 51, 522-527 (2017).

30. Sun, $H$. et al. $\beta 7$ integrin inhibition can increase intestinal inflammation by impairing homing of CD25(hi)FoxP3(+) regulatory T cells. Cell. Mol. Gastroenterol. Hepatol. https://doi.org/10.1016/j.jcmgh.2019.10.012 (2019).

31. Jaensson, E. et al. Small intestinal CD103+ dendritic cells display unique functional properties that are conserved between mice and humans. J. Exp. Med. 205, 2139-2149 (2008).

32. Fagarasan, S. et al. Critical roles of activation-induced cytidine deaminase in the homeostasis of gut flora. Science 298, 1424-1427 (2002).

33. Babbar, A. et al. The compromised mucosal immune system of beta7 integrindeficient mice has only minor effects on the fecal microbiota in homeostasis. Front. Microbiol. 10, 2284 (2019).

34. Maharshak, N. et al. Altered enteric microbiota ecology in interleukin 10-deficient mice during development and progression of intestinal inflammation. Gut Microbes 4, 316-324 (2013).

35. Gorfu, G., Rivera-Nieves, J. \& Ley, K. Role of beta7 integrins in intestinal lymphocyte homing and retention. Curr. Mol. Med. 9, 836-850 (2009).

36. Davidson, N. J. et al. T helper cell 1-type CD4+ T cells, but not B cells, mediate colitis in interleukin 10-deficient mice. J. Exp. Med. 184, 241-251 (1996).

37. Bjursten, M., Bland, P. W., Willen, R. \& Hornquist, E. H. Long-term treatment with anti-alpha 4 integrin antibodies aggravates colitis in $\mathrm{G}$ alpha i2-deficient mice. Eur. J. Immunol. 35, 2274-2283 (2005).

38. Brynjolfsson, S. F. et al. Long-lived plasma cells in mice and men. Front. Immunol. 9, 2673 (2018).

39. Sandborn, W. J. The present and future of inflammatory bowel disease treatment. Gastroenterol. Hepatol. 12, 438-441 (2016).

40. Leiper, K. et al. Randomised placebo-controlled trial of rituximab (anti-CD20) in active ulcerative colitis. Gut 60, 1520-1526 (2011).

41. Uzzan, M. et al. Anti-alpha4beta7 therapy targets lymphoid aggregates in the gastrointestinal tract of HIV-1-infected individuals. Sci. Transl. Med. 10, eaau4711 https://doi.org/10.1126/scitranslmed.aau4711 (2018).

42. Verstockt, B. et al. Expression levels of 4 genes in colon tissue might be used to predict which patients will enter endoscopic remission after vedolizumab therapy for inflammatory bowel diseases. Clin. Gastroenterol. Hepatol. 18, 1142-1151 (2020). e1110.

43. Wyant, T. et al. Vedolizumab affects antibody responses to immunisation selec tively in the gastrointestinal tract: randomised controlled trial results. Gut $\mathbf{6 4}$ 77-83 (2015).

44. Habtezion, A., Nguyen, L. P., Hadeiba, H. \& Butcher, E. C. Leukocyte trafficking to the small intestine and colon. Gastroenterology 150, 340-354 (2016).

45. Schippers, A. et al. beta7 integrin controls immunogenic and tolerogenic mucosal B cell responses. Clin. Immunol. 144, 87-97 (2012).

46. Allner, $C$. et al. Baseline levels of dynamic CD4(+) T cell adhesion to MAdCAM-1 correlate with clinical response to vedolizumab treatment in ulcerative colitis: a cohort study. BMC Gastroenterol. 20, 103 (2020).

47. Hassan-Zahraee, M. et al. Anti-MAdCAM antibody increases ss7+ T cells and CCR9 gene expression in the peripheral blood of patients with Crohn's disease. J. Crohn's Colitis 12, 77-86 (2018).

48. Harriman, G. R. et al. Targeted deletion of the IgA constant region in mice leads to IgA deficiency with alterations in expression of other Ig isotypes. J. Immunol. 162, 2521-2529 (1999).
49. Tyler, C. J. et al. Implementation of mass cytometry as a tool for mechanism of action studies in inflammatory bowel disease. Inflamm. Bowel Dis. 24, 2366-2376 (2018).

50. Nowicka, M. et al. CyTOF workflow: differential discovery in high-throughput high-dimensional cytometry datasets. F1000Res. 6, 748 (2017).

\section{ACKNOWLEDGEMENTS}

The authors thank Michael Solga of the University of Virginia Flow Cytometry Core for assistance with mass cytometry event acquisition.

\section{AUTHOR CONTRIBUTIONS}

C.J.T. and J.R.-N.: study concept and design and analysis and interpretation of data; C.J.T., M.G., L.R.L., S.Y., N.Z., and P.J.: sample processing and data acquisition; C.J.T., G. B., and J.R.-N.: drafting of manuscript; and G.B.: critical revision of manuscript.

\section{FUNDING INFORMATION}

This work was supported by grants from the National Institutes of Health [DK108670, DK118927]; VA Merit BLRD-I01 BX003436; Takeda Pharmaceuticals U.S.A. IISR-2017102050 grant to J.R.-N.; San Diego Digestive Diseases Research Center [P30 DK120515]; Shared Instrumentation Grant [SIG] Program [S10]: CyTOF Mass Cytometer S10 OD018499-01 to La Jolla Institute of Allergy and Immunology (L.J.I.); and Chiba University-UC San Diego Program in Mucosal Immunology, Allergy and Vaccines.

\section{COMPETING INTERESTS}

The authors declare no competing interests.

\section{ADDITIONAL INFORMATION}

Supplementary information The online version contains supplementary material available at https://doi.org/10.1038/s41385-021-00445-z.

Correspondence and requests for materials should be addressed to J.R-N.

Reprints and permission information is available at http://www.nature.com/ reprints

Publisher's note Springer Nature remains neutral with regard to jurisdictional claims in published maps and institutional affiliations.

Open Access This article is licensed under a Creative Commons Attribution 4.0 International License, which permits use, sharing, adaptation, distribution and reproduction in any medium or format, as long as you give appropriate credit to the original author(s) and the source, provide a link to the Creative Commons license, and indicate if changes were made. The images or other third party material in this article are included in the article's Creative Commons license, unless indicated otherwise in a credit line to the material. If material is not included in the article's Creative Commons license and your intended use is not permitted by statutory regulation or exceeds the permitted use, you will need to obtain permission directly from the copyright holder. To view a copy of this license, visit http://creativecommons. org/licenses/by/4.0/.

This is a U.S. government work and not under copyright protection in the U.S.; foreign copyright protection may apply 2021 